\title{
Article \\ Multiple Dielectric-Supported Ridge-Loaded Rhombus-Shaped Wideband Meander-Line Slow-Wave Structure for a V-Band TWT
}

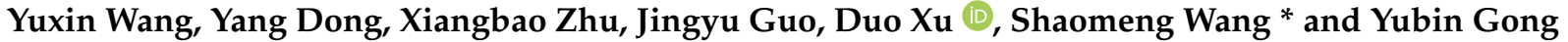

Citation: Wang, Y.; Dong, Y.; Zhu, X.; Guo, J.; Xu, D.; Wang, S.; Gong, Y. Multiple Dielectric-Supported Ridge-Loaded Rhombus-Shaped Wideband Meander-Line Slow-Wave Structure for a V-Band TWT. Electronics 2022, 11, 405. https:// doi.org/10.3390/electronics11030405

Academic Editor: Je-Hyeong Bahk

Received: 10 December 2021

Accepted: 26 January 2022

Published: 28 January 2022

Publisher's Note: MDPI stays neutral with regard to jurisdictional claims in published maps and institutional affiliations.

Copyright: (C) 2022 by the authors. Licensee MDPI, Basel, Switzerland. This article is an open access article distributed under the terms and conditions of the Creative Commons Attribution (CC BY) license (https:// creativecommons.org/licenses/by/ $4.0 /)$
National Key Laboratory of Science and Technology on Vacuum Electronics, University of Electronic Science and Technology of China, Chengdu 610054, China; 202022022426@std.uestc.edu.cn (Y.W.); 202111022405@std.uestc.edu.cn (Y.D.); 202022021934@std.uestc.edu.cn (X.Z.); 202111022436@std.uestc.edu.cn (J.G.); xuduo1234567@hotmail.com (D.X.); ybgong@uestc.edu.cn (Y.G.)

* Correspondence: wangsm@uestc.edu.cn; Tel.: +86-2883201538

\begin{abstract}
A multiple dielectric-supported ridge-loaded rhombus-shaped meander-line (MDSRLRSML) slow-wave structure (SWS) is proposed for a V-band wideband traveling wave tube (TWT). The high-frequency and transmission characteristics of the SWS are investigated. The proposed structure can realize stable output via attenuator and special phase-velocity jumping. Particle-in-cell (PIC) results indicate that, for a $7 \mathrm{kV}, 0.1 \mathrm{~A}$ sheet-beam, the average output power can reach $60 \mathrm{~W}$ at $60 \mathrm{GHz}$ and a $3 \mathrm{~dB}$ bandwidth of $9 \mathrm{GHz}$, with the corresponding gain and electron efficiency of $30.8 \mathrm{~dB}$ and $17.2 \%$, respectively. Compared with the dielectric-supported rhombus-shape meanderline (DS-RSML) SWS, the proposed structure has a wider bandwidth, higher gain, more stable structure, and better heat dissipation ability, which make it a good candidate source in millimeterwave communications.
\end{abstract}

Keywords: meander-line; phase-velocity jumping; thermal analysis; slow-wave structure; traveling wave tube

\section{Introduction}

The traveling wave tube (TWT) is an important type of vacuum electronic device, which is widely used in radar, satellite communication, medical imaging electronic countermeasures, and other fields because of its excellent characteristics of high power, wide band, low noise, and high gain [1]. The traditional TWTs mainly include helix TWTs and coupled cavity TWTs. However, when the operation frequency is increased to the millimeter-wave or terahertz band, the traditional helix traveling wave tube faces great challenges during fabrication due to its small size [2]. At this time, there is an urgent demand for high-efficiency and high-power TWTs, and the planar TWT which is compatible with higher-precision microelectronics machine system (MEMS) technology has become a new research hotpot [3-5]. The planar TWT mainly refers to planar slow-wave structures (SWSs), including rectangular spiral SWSs [6-9], microstrip meander-line SWSs [10-12], staggered double-gate SWSs [13,14], and folded waveguide (FWG) SWSs [15].

Microstrip meander-line SWSs have been studied intensively for years, because of their advantages of easy integration, easy processing, and suitability for sheet-beam operation [16-19]. For instance, a V-band microstrip meander-line SWS, which was proposed in [19], could achieve rapid production using magnetron sputtering and laser ablation micromachining techniques. However, this kind of traditional microstrip meander-line usually uses a dielectric substrate to support the SWS, which is not resistant to electron bombardment and has the problem of charge accumulation and short-circuit.

In order to solve these problems, a new type of meander-line supported by dielectric rods was proposed in [20-23], in which the dielectric substrate was replaced by side 
rods. The so-called dielectric-rod-supported metallic meander-line SWS is suitable for a dual-sheet electron beam, thus having large power capability. For example, the Ka-band meander-line SWS supported by dielectric rods mentioned in [21] can achieve a gain of $23.4 \mathrm{~dB}$ at a voltage of $10.6 \mathrm{kV}$ and a bandwidth of $6 \mathrm{GHz}$; the Ka-band ring-bar SWS supported by dielectric rods proposed in [22] can achieve a gain of $22.6 \mathrm{~dB}$ at $9.7 \mathrm{kV}$ and a bandwidth of $2 \mathrm{GHz}$.

A dual-beam rhombus-shaped meander-line (DS-RSML) SWS supported from both sides by dielectric rods was proposed in [24], as shown in Figure 1a. This SWS can operate at a higher frequency with a wider lateral dimension compared to the dielectric-rod-supported metallic meander-line SWS. The particle-in-cell simulation showed that it can reach $83 \mathrm{~W}$ average output power at $58 \mathrm{GHz}$ with a sheet electron beam of $7.7 \mathrm{kV}, 0.1 \mathrm{~A}$. However, its $3 \mathrm{~dB}$ bandwidth was only $6.5 \mathrm{GHz}$, and it struggled with heat dissipation. It was found that the ridge loading method can effectively broaden the bandwidth and reduce the operating voltage of the microstrip meander line [25]. Therefore, a multiple dielectric-supported ridge-loaded rhombus-shaped meander-line (MDSRL-RSML) SWS is proposed in this paper, as shown in Figure 1b.

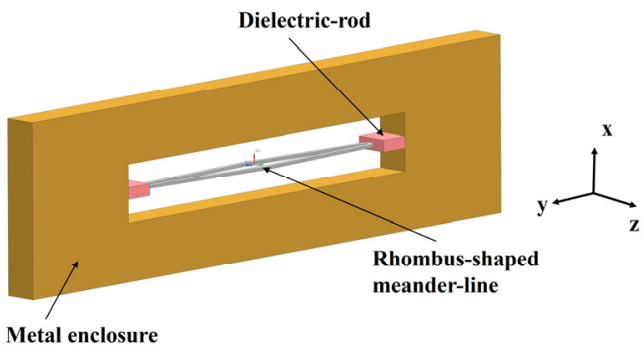

(a)

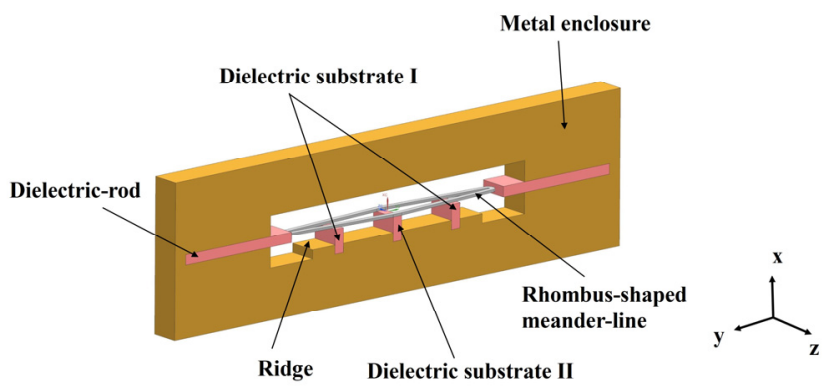

(b)

Figure 1. Single period of (a) DS-RSML SWS and (b) MDSRL-RSML SWS models.

The proposed structure of the DS-RSML SWS weakens the dispersion intensity of the TWT by employing metal ridges on the inner surface of the metal shell, so as to reduce the operation voltage and improve the working bandwidth of the TWT. Meanwhile, multiple dielectric rods embedded in the metal shell and ridge are applied on both sides and the bottom of the meander line, effectively solving the heat dissipation problem of the SWS and making the structure more stable. In addition, upon increasing the thickness of the dielectric substrate I along the propagation direction, the structural phase velocity is changed, which suppresses the backward wave oscillation and reduces the design difficulty. A glossary of abbreviations in the article is presented in Table 1.

Table 1. Glossary of abbreviations.

\begin{tabular}{cc}
\hline Abbreviations & The Full Name \\
\hline SWS & Slow-wave structure \\
\hline TWT & Traveling wave tube \\
\hline MDSRL-RSML & $\begin{array}{c}\text { Multiple dielectric-supported ridge-loaded } \\
\text { rhombus-shaped meander-line }\end{array}$ \\
\hline DS-RSML & Dielectric-supported rhombus-shape meander line \\
PIC & Particle-in-cell \\
MEMS & Microelectronics machine system \\
FWG & Folded waveguide \\
ML & Meander-line \\
\hline
\end{tabular}

The structure design and settings of the MDSRL-RSML, including the eigenmode structure, input and output coupling structure, and thermal analysis structure, are described 
in Section 2. Section 3 presents the high-frequency characteristics, cold and hot simulation results, and thermal analysis results. In Section 4, the results are analyzed and discussed. A short conclusion is presented in Section 5.

\section{Materials and Structures}

\subsection{Single-Period Model of the MDSRL-RSML TWT}

As shown in Figure 1b, the proposed structure consists of a metal enclosure with a ridge on the bottom edge, a rhombus-shaped meander-line, a pair of side supporting rods, and three bottom supporting dielectric rods. Taking processing into consideration, it is easier to achieve the clamping between the dielectric rods and the meander-line, as well as the connection between different periods of the meander-line, using a circular arc or straight line instead of inner and outer corners.

In the simulation, the material of the ridge-loaded metal enclosure and the rhombusshaped meander-line is copper with a conductivity of $3 \times 10^{7} \mathrm{~S} / \mathrm{m}$, which was achieved by magnetron sputtering on a molybdenum surface (conductivity of $2 \times 10^{7} \mathrm{~S} / \mathrm{m}$ ) with a $3 \mu \mathrm{m}$ thick copper layer (conductivity of $5.8 \times 10^{7} \mathrm{~S} / \mathrm{m}$ ) [26]. The dielectric rods are made from boron nitride (BN, relative dielectric constant of 5.12 and loss tangent of 0.005$)$. The side supporting dielectric rods mainly play the role of clamping and stabilization. The bottom supporting dielectric rods are fixed in the ridge, supporting the meander-line from bottom up; they are used for support, phase-velocity taper, and heat dissipation.

The configuration of a single period of the proposed SWS with labeled dimensional parameters is presented in Figure 2a, and the specific shape of the rhombus-shaped meanderline is given in Figure $2 b$.

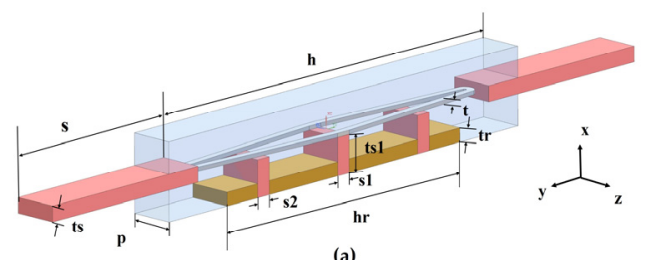

(a)

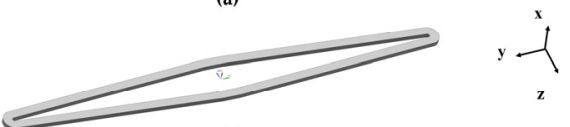

(b)

Figure 2. (a) Dimensional parameters of the MDSRL-RSML SWS; (b) rhombus-shaped meander-line.

The periodic length and the transverse length of the meander-line are represented by $p$ and $\mathrm{h}$, respectively. Table 2 shows the main dimensional parameters of the MDSRL-RSML SWS.

Table 2. Optimized dimensional parameters of the MDSRL-RSML SWS.

\begin{tabular}{cccc}
\hline Parameters & Value $(\mathbf{m m})$ & Parameters & Value (mm) \\
\hline $\mathrm{h}$ & 2 & $\mathrm{~s}$ & 1 \\
$\mathrm{t}$ & 0.02 & $\mathrm{~s} 1$ & 0.078 \\
$\mathrm{p}$ & 0.23 & $\mathrm{~s} 2$ & 0.08 \\
$\mathrm{hr}$ & 1.6 & $\mathrm{ts}$ & 0.08 \\
$\mathrm{tr}$ & 0.07 & $\mathrm{ts} 1$ & 0.19 \\
\hline
\end{tabular}

\subsection{Complete Transmission and Interaction Model of the MDSRL-RSML TWT}

Figure 3 a shows a schematic diagram of the overall MDSRL-RSML SWS. The entire SWS can be divided into five segments exclusive of the input and output parts, and each segment adopts different s2 parameters (the thickness of the dielectric rods I) to suppress the backward-wave oscillation of the structure instead of pitch tapering. In addition, the geometry of the attenuator is described in Figure 3b, where the two dielectric rods and dielectric rods II are coated with attenuating material for a 15-period length, while the material of the attenuator is beryllium oxide with a dielectric constant of 6.5 and loss 
tangent of 0.5. In Figure 3c, the structures at both ends of the slow-wave line include three periods of gradient with a height ratio of $R$ to reduce reflection. Furthermore, in order to connect the meander-line SWS to a WR-15 rectangular waveguide port with reduced reflection, a stepped single-ridge waveguide is used, as shown in Figure 3d.

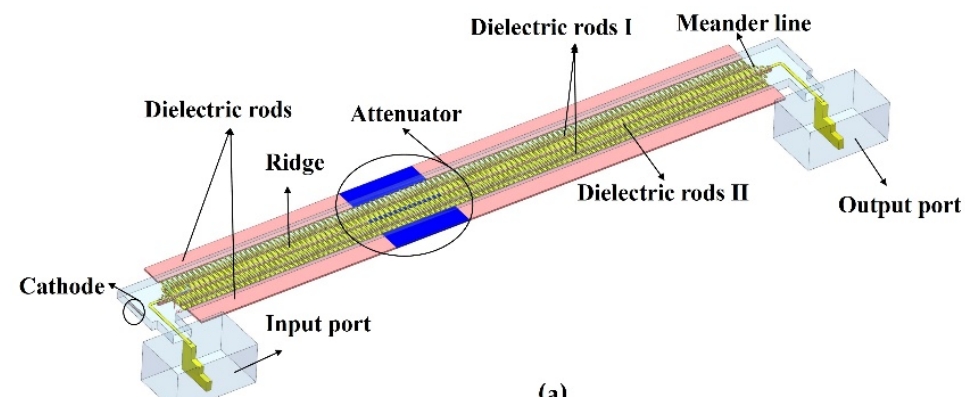

(a)

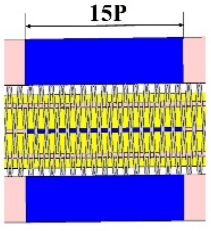

(b)

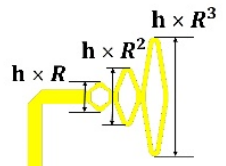

(c)

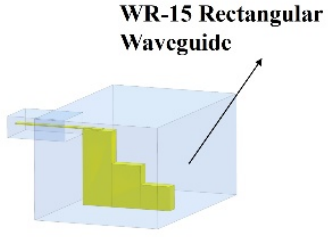

(d)

Figure 3. (a) The whole MDSRL-RSML SWS structure; (b) attenuator section; (c) gradient section; (d) the input-output couplers of the stepped ridge waveguide transition.

The optimized thickness of the dielectric rod I (s2) varies with the number of axial periods as shown in Figure 4.

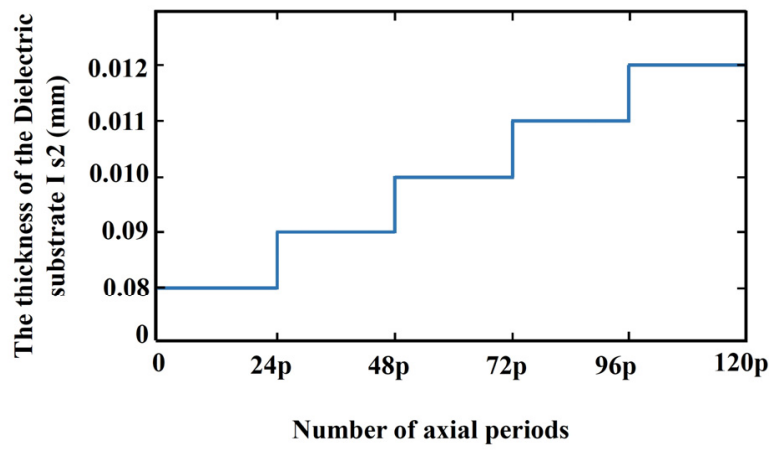

Figure 4. The thickness of the dielectric rod I (s2) as a function of the axial period.

\subsection{Thermal Analysis Model of the MDSRL-RSML TWT}

The tapered-S2 SWS with a length of $29.97 \mathrm{~mm}$ was used for the thermal analysis. The background temperature was $300 \mathrm{~K}$, while the thermal conductivity of the material was as described in Table 3.

Table 3. Material properties in the simulation.

\begin{tabular}{cc}
\hline Material & Thermal Conductivity (W/K/m) \\
\hline Copper & 401 \\
BN & 60 \\
BEO & 250 \\
\hline
\end{tabular}

In order to calculate thermal loss, we divided the whole SWS into 17 sections. The input and output parts were in one section each, with a length of $1.185 \mathrm{~mm}$. The middle part contained 15 sections; the attenuator part had a length of $3.45 \mathrm{~mm}, 13$ sections all had 
a length of $2.3 \mathrm{~mm}$, and the last section had a length of $1.15 \mathrm{~mm}$. The power flow curve in the PIC simulation was segmented to calculate the transmitted power of each part. Since $\mathrm{S} 21$ was $-12.07 \mathrm{~dB}$ at $60 \mathrm{GHz}$ without an attenuator, its section was about $0.4027 \mathrm{~dB} / \mathrm{mm}$. The power loss of each section could be calculated as follows [27]:

$$
P_{\text {loss }}=\frac{P_{\text {transmitted }}}{10^{0.1 \times S_{21 \_s e c t i o n}}}-P_{\text {transmitted }} \text {. }
$$

The calculated power loss for each section is listed in Table 4 with a total loss of $35.34 \mathrm{~W}$. Then, the power lost in each segment was set to be the heat source, and the shell was set to convection with a convective coefficient of $50 \mathrm{~W} / \mathrm{mm}^{2} \cdot \mathrm{K}$.

Table 4. Power loss for each section.

\begin{tabular}{cccccccc}
\hline No. & $\mathbf{1}$ & $\mathbf{2}$ & $\mathbf{3}$ & $\mathbf{4}$ & $\mathbf{5}$ & $\mathbf{6}$ & $\mathbf{7}$ \\
\hline$P_{\text {loss }}(\mathrm{W})$ & 0.0139 & 0.0241 & 0.0261 & 0.0491 & 0.1177 & 0.1855 & 0.2456 \\
No. & 8 & 9 & 10 & 11 & 12 & 13 & 14 \\
$P_{\text {loss }}(\mathrm{W})$ & 0.7748 & 1.8581 & 3.9044 & 7.8145 & 13.4085 & 6.7522 & 0.1656 \\
\hline
\end{tabular}

\section{Simulation Results}

\subsection{High-Frequency Characteristics of the MDSRL-RSML TWT}

The CST Eigenmode Solver was adopted to obtain the high-frequency characteristics of the MDSRL-RSML fundamental mode. For the dimension values in Table 2, the dispersion and coupling impedance curves of the DS-RSML and MDSRL-RSML are shown in Figure 5a,b. Although the former structure showed a higher coupling impedance in the low-frequency band, the latter structure had a lower normalized phase velocity and flatter dispersion characteristics, indicating a lower operating voltage and broader cold bandwidth, respectively.

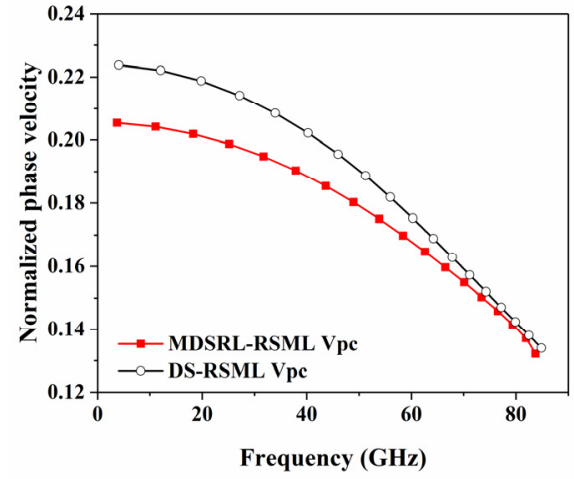

(a)

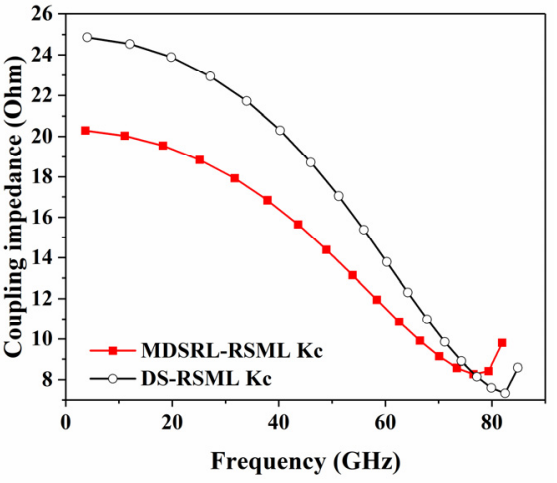

(b)

Figure 5. Comparison of (a) dispersion and (b) coupling impedance characteristics between DS-RSML and MDSRL-RSML SWS.

Figure 6 shows the electric field axial-component distributions of the fundamental mode and the higher-order mode along the transverse direction, both of which were strong in the middle and weak at both ends, indicating that the electric fields were suitable for the work of sheet-beam. However, this also suggests that, when the position of the electron beam was in the middle, the higher-order mode could compete with the fundamental mode. 


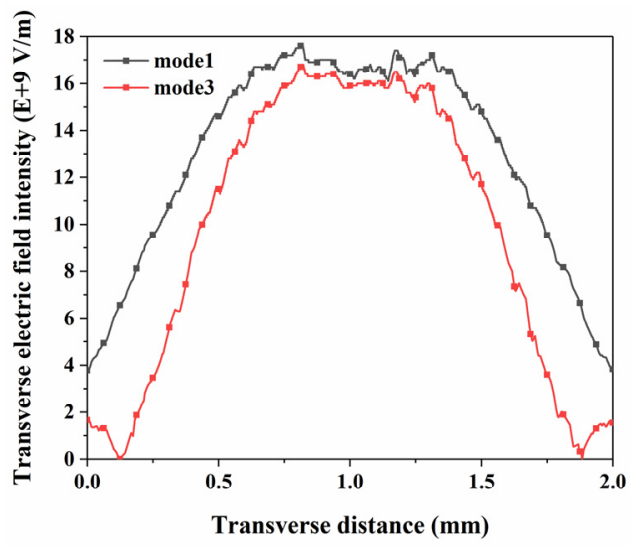

Figure 6. Transverse electric field distribution of fundamental mode and higher-order mode.

Figure $7 \mathrm{a}, \mathrm{b}$ show the $\omega-\beta$ diagram and normalized phase velocity for the different values of s2, respectively. When s2 $=0.08 \mathrm{~mm}$, the beam line, as shown in Figure $7 \mathrm{a}$, intersected with each eigenmode at the following frequencies: mode 1 in the forward-wave region of $60 \mathrm{GHz}$, which could realize wave amplification; the intersection points with the higher-order modes 3 and 5 were located at $93 \mathrm{GHz}$ and $175 \mathrm{GHz}$, respectively, in the backward-wave region, facilitating backward-wave oscillations.

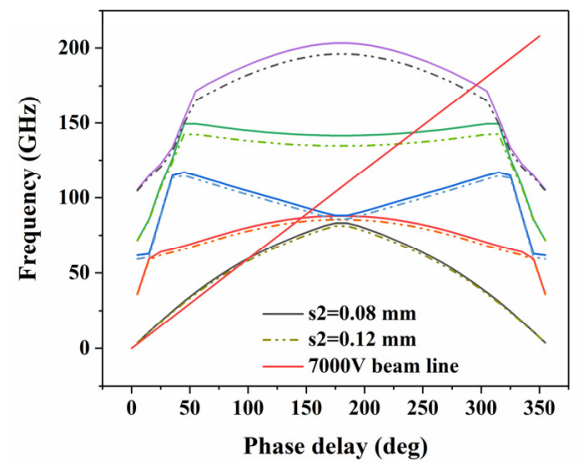

(a)

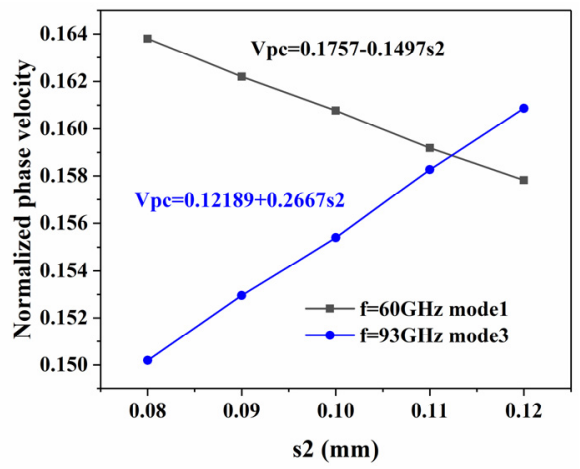

(b)

Figure 7. (a) $\omega$ - $\beta$ diagram and (b) normalized phase-velocity characteristics with different s2 (in $\mathrm{mm}$ ).

In Figure 7a, it can also be clearly seen that, as s2 increased, the eigenfrequency of the fundamental mode decreased much more slowly than the higher-order modes, which could effectively separate the operation voltages between the fundamental mode and higher-order modes and destroy their synchronization. Figure $7 \mathrm{~b}$ gives a more intuitive description; as s2 changed, the normalized phase velocity of higher-order modes became more sensitive, and the corresponding voltage changed more than the fundamental mode. As a result, changing the rod width can be used as an effective method to suppress backward-wave oscillation.

\subsection{Transmission Characteristics of the MDSRL-RSML TWT}

The transmission characteristics of the 120-period MDSRL-RSML SWS together with the input-output couplers were determined using CST Microwave Studio [28]. The simulation results with and without attenuator are compared in Figure 8. Obviously, the attenuator only slightly changed $\mathrm{S} 11$, which was below $-15 \mathrm{~dB}$ over the frequency range of 54-65 GHz, while S21 decreased about $-15 \mathrm{~dB}$ across the whole frequency band, indicating that the attenuator effectively absorbed the wave energy. 


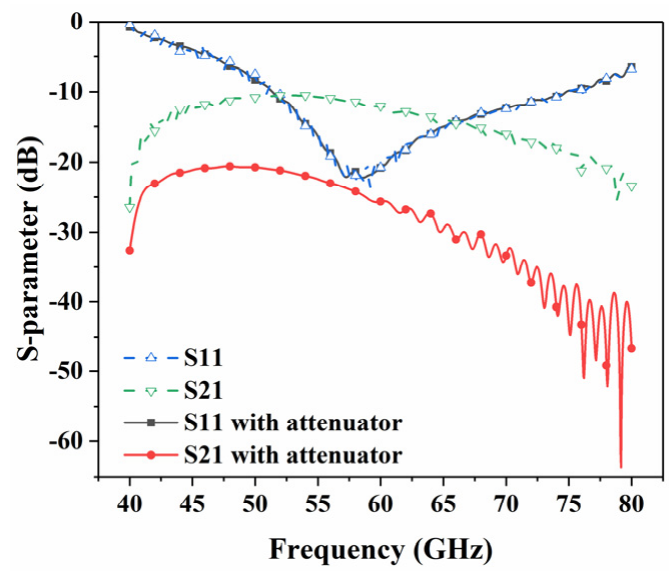

Figure 8. The S-parameters of the MDSRL-RSML SWS.

\section{3. "Hot" Performance of the MDSRL-RSML TWT}

In order to study the "hot" performance of the MDSRL-RSML SWS TWT, PIC simulations were conducted using CST Particle Studio [28]. An electron beam with a voltage of $7000 \mathrm{~V}$ and current of $0.1 \mathrm{~A}$ (current density of $143 \mathrm{~A} / \mathrm{cm}^{2}$ ) was employed, and the height and width of the sheet-beam were $1 \mathrm{~mm}$ and $0.07 \mathrm{~mm}$, respectively. In addition, a solenoid focusing magnetic field of $0.6 \mathrm{~T}$ was used to maintain the beam shape, and the number of periods of the TWT was 120 with a whole length of $31 \mathrm{~mm}$.

For an input signal of $0.05 \mathrm{~W}$, the corresponding signal diagram and spectrum diagram at $60 \mathrm{GHz}$ were obtained as shown in Figure 9a,b, respectively. Due to the combination of attenuator and phase-velocity jumping techniques, a stable output was achieved, and the signal was effectively amplified. Meanwhile, it can be seen from Figure $9 \mathrm{~b}$ that the spectrum was pure; the harmonic components were about $-50 \mathrm{~dB}$ smaller than the fundamental frequency, and the oscillation risk was effectively suppressed.

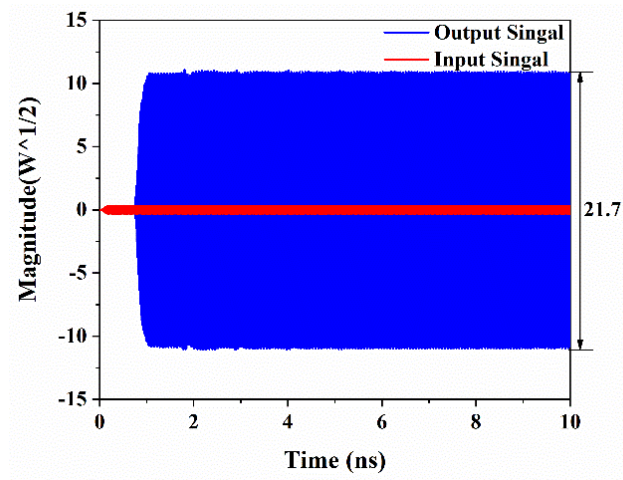

(a)

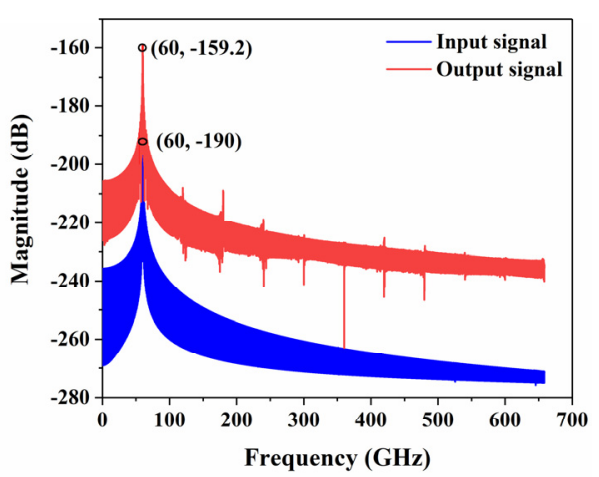

(b)

Figure 9. (a) Variation of input and output signals with time; (b) Fourier spectrum diagram of the output signal.

Figure 10 shows the electron beam bunching, as well as the phase space diagram of the electron beam, with most particles losing energy and a few electrons gaining energy, showing a sufficient beam wave energy transfer. 


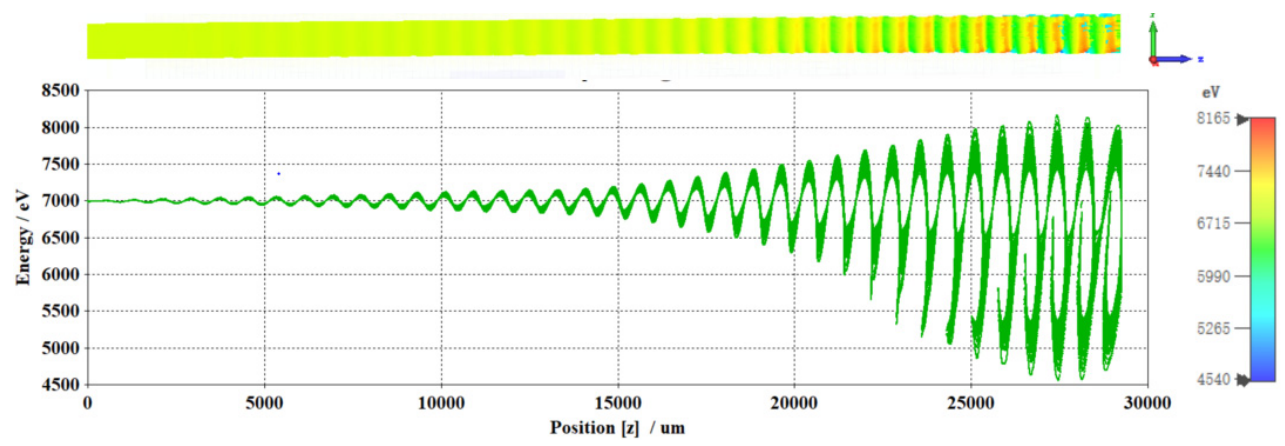

Figure 10. Phase space diagram.

The variation of average output powers of the DS-RSML and MDSRL-RSML at $60 \mathrm{GHz}$ with the input power is depicted in Figure 11a,b. It can be seen that the maximum average output power of the former was $83 \mathrm{~W}$ at $0.17 \mathrm{~W}$ input power, with the corresponding saturation gain of $27 \mathrm{~dB}$, while the saturation input power of the latter was reduced to $0.05 \mathrm{~W}$, with the corresponding gain of $30.8 \mathrm{~dB}$. Since the simulation was performed at the optimal operating voltages of both structures, the reduction in output power was to be expected. The former structure had an operating working voltage of $7700 \mathrm{~V}$, while the optimal operating voltage of the latter structure was reduced to $7000 \mathrm{~V}$.

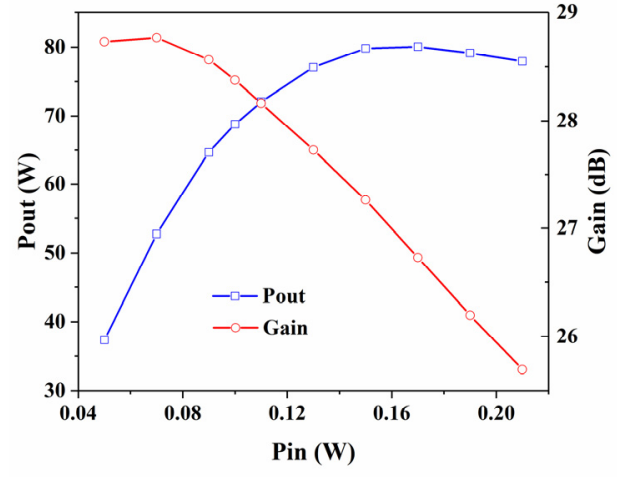

(a)

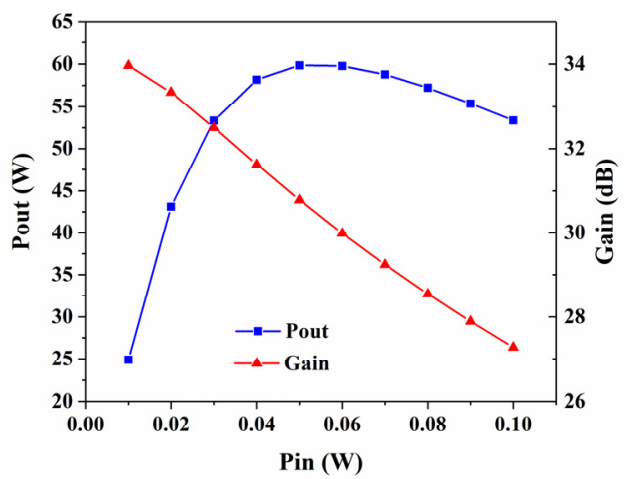

(b)

Figure 11. Variation of average output power and gain at $60 \mathrm{GHz}$ with input power for the (a) DSRSML SWS and (b) MDSRL-RSML SWS.

A comparison of DS-RSML and MDSRL-RSML in terms of the average output power and gain versus frequency is shown in Figure 12a,b. When the voltage was $7000 \mathrm{~V}$, the maximum average output power of the MDSRL-RSML structure was $60 \mathrm{~W}$ at a frequency of $60 \mathrm{GHz}$, with an average gain of $30.8 \mathrm{~dB}$ and a $3 \mathrm{~dB}$ bandwidth of $9 \mathrm{GHz}(53.5-62.5 \mathrm{GHz})$. The electron efficiency was $17.2 \%$. The $3 \mathrm{~dB}$ bandwidth of MDSRL-RSML was about 1.5 times that of the DS-RSML; although the output power was reduced compared with the DS-RSML, it could achieve a higher gain. 


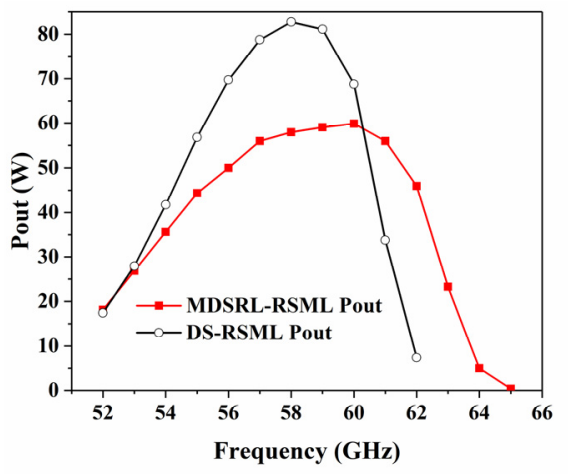

(a)

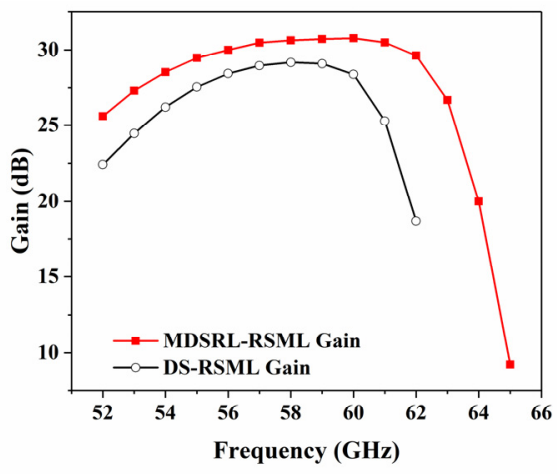

(b)

Figure 12. (a) Average output power and (b) gain versus frequency for the DS-RSML SWS and MDSRL-RSML SWS.

\subsection{Thermal Distribution of the MDSRL-RSML TWT}

For a traveling wave tube, good heat dissipation capability is a necessary condition for normal operation. To verify the heat-handling capacity of the proposed SWS, thermal analysis [29] of these two structures was performed using the CST thermal solver with the structure and settings described in Section 2.3. Here, we assumed that the flow rate of the electron beam was $100 \%$, and only the heat loss due to ohmic loss was considered. Meanwhile, to simplify the thermal analysis, the thermal contact resistance (TCR) between different materials was not taken into consideration.

Figure 13 shows the temperature distribution of the two structures. In the DS-RSML, the maximum temperature distribution of each section was in the center of the meander line, because this section did not come into contact with the dielectric rods; thus, the maximum temperature could reach about $793.85^{\circ} \mathrm{C}$. On the other hand, the MDSRL-RSML had several supporting rods to help heat dissipation; thus, the maximum temperature could be maintained at $130.85^{\circ} \mathrm{C}$.

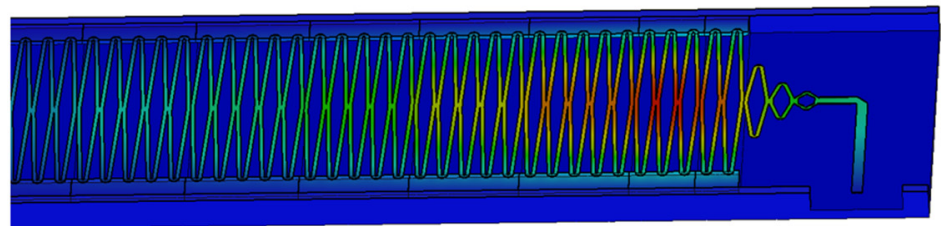

(a)

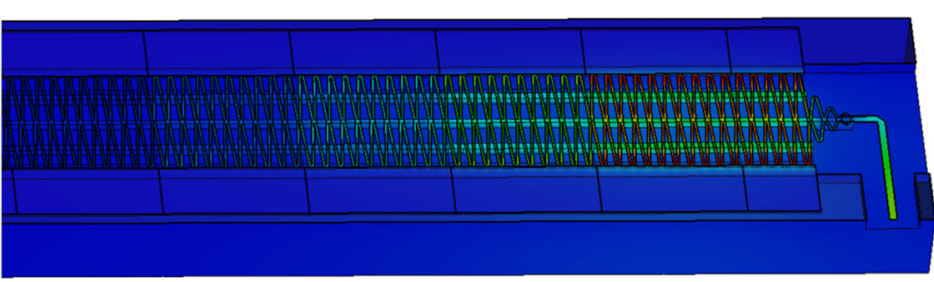

(b)
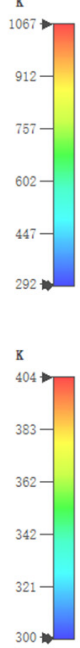

Figure 13. The maximum temperature distribution in the (a) DS-RSML SWS and (b) MDSRLRSML SWS.

Figure 14 shows the temperature distribution along the axis of the DS-RSML SWS and MDSRL-RSML SWS. The proposed structure had better heat dissipation characteristics; accordingly, it would not suffer from excessively high temperature under a highfrequency power of tens of watts, effectively solving the heat dissipation problem in the previous structure. 


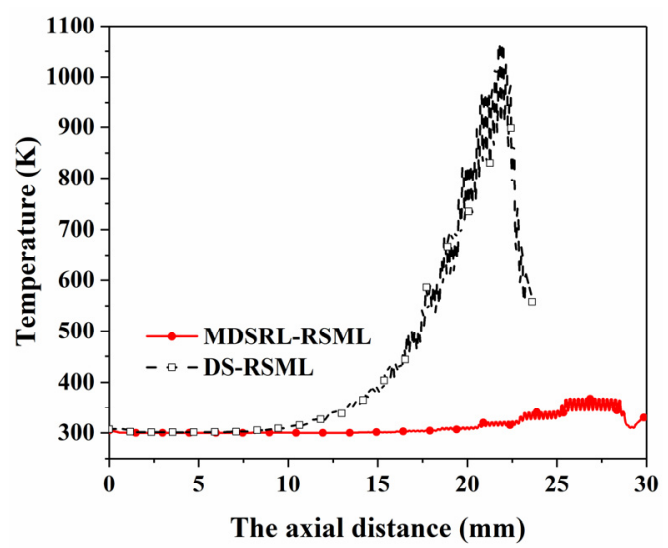

Figure 14. Temperature distribution along the axis of the DS-RSML SWS and MDSRL-RSML SWS.

\section{Discussions}

From the results presented in Section 3, it can be seen that the MDSRL-RSML SWS has the advantages of wide bandwidth and high gain. Furthermore, it offers a special method to suppress the backward-wave oscillation. Additionally, the problems of heat dissipation and stability of the SWS are solved in this structure.

Table 5 lists the key operation parameters of meander-line SWSs in the literature, as well as those of the proposed SWS; as it can be seen, compared with the specific parameters of the meander-line SWSs supported by dielectric rods [21,22,26], the gain of the proposed structure can exceed $30 \mathrm{~dB}$ while keeping a miniaturized structure. Compared with the structure in [18], the proposed structure has a lower operating voltage while maintaining a wide relative bandwidth. Compared with the structures in $[19,23]$, the relative bandwidth of MSDRL-RSML was 2-3 times higher at a low operating voltage.

Table 5. Performance comparison of dielectric-supported meander-line SWSs.

\begin{tabular}{cccccc}
\hline Model & $\begin{array}{c}\text { Frequency } \\
\text { Band }\end{array}$ & $\begin{array}{c}\text { Voltage } \\
(\mathbf{k V})\end{array}$ & $\begin{array}{c}\text { Gain } \\
(\mathbf{d B})\end{array}$ & $\begin{array}{c}\text { Bandwidth } \\
\text { (GHz) }\end{array}$ & $\begin{array}{c}\text { Relative } \\
\text { Bandwidth }\end{array}$ \\
\hline$[21]$ & $\mathrm{Ka}$ & 10.6 & 23.4 & 6 & $15.8 \%$ \\
{$[22]$} & $\mathrm{Ka}$ & 9.7 & 22.6 & 2 & $5.3 \%$ \\
{$[26]$} & $\mathrm{Ka}$ & 4.4 & 24.1 & 2.5 & $6.6 \%$ \\
MDSRL-RSML & $\mathrm{V}$ & 7 & 30.8 & 9 & $15 \%$ \\
\hline
\end{tabular}

In addition, the main results presented in Section 3 were correlated using equations in MATLAB. For instance, the normalized phase velocity $(V p c)$ and s2 of mode $1\left(V_{p c 1}\right)$ and mode $3\left(V_{p c 3}\right)$ in Figure $7 \mathrm{~b}$ approximately satisfied the following relationships:

$$
\begin{gathered}
V_{p c 1}=0.1757-0.1497 s_{2}, \\
V_{p c 3}=0.12189+0.2667 s_{2} .
\end{gathered}
$$

As shown in Figure 11a, the relationship of output power and gain with input power of the DS-RSML structure could be approximately expressed as follows:

$$
\begin{aligned}
& P_{\text {out }}=-23.84+1579 P_{i n}-7727 P_{i n}^{2}+11970 P_{i n}^{3}, \\
& \text { Gain }=27.79+36.65 P_{i n}-384.8 P_{i n}^{2}+776.4 P_{i n}^{3} .
\end{aligned}
$$

As shown in Figure 11b, the relationship of output power and gain with input power of the MDSRL-RSML structure could be approximately expressed as follows:

$$
\begin{aligned}
& P_{\text {out }}=-74.59+2958 P_{i n}-22900 P_{i n}^{2}+73410 P_{i n}^{3}-83830 P_{i n}^{4}, \\
& \text { Gain }=34.33+329.2 P_{i n}^{2}-13180 P_{i n}^{3}+9180 P_{i n}^{4}-187700 P_{i n}^{5} .
\end{aligned}
$$




\section{Conclusions}

An MDSRL-RSML SWS for V-band TWTs was proposed and investigated in this paper. The dispersion characteristics and coupling impedance were studied, revealing that the proposed structure has flatter dispersion characteristics and a lower beam voltage than DS-RSML. The proposed structure has good transmission characteristics, with S11 below $-15 \mathrm{~dB}$ over the frequency range of $54-65 \mathrm{GHz}$. Stable output can be realized by adding an attenuator and using special phase-velocity jumping. The PIC result indicated a maximum average output power of $60 \mathrm{~W}$ at $60 \mathrm{GHz}$ for a beam voltage of $7000 \mathrm{~V}$ and beam current of 0.1 A with a bandwidth of $9 \mathrm{GHz}$, with a corresponding gain and electron efficiency of $30.8 \mathrm{~dB}$ and $17.2 \%$, respectively. Compared with DS-RSML, the MDSRL-RSML has a wider bandwidth and higher gain; furthermore, this structure solves the heat dissipation problem and improves the stability of the whole structure.

Author Contributions: Conceptualization, Y.W.; data curation, X.Z.; project administration, S.W.; Supervision, Y.G.; Validation, Y.D.; Writing—original draft, J.G.; Writing—review \& editing, D.X. All authors have read and agreed to the published version of the manuscript.

Funding: This work was supported by the National Natural Science Foundation of China (NSFC) (Grant Nos. 61921002, 92163204, 61988102) and the Laboratory Fund of the National Key Lab on Microwave Vacuum Devices (Grant No. 19ZS202807200303A).

Conflicts of Interest: The authors declare no conflict of interest.

\section{References}

1. Chong, C.K.; Menninger, W.L. Latest Advancements in High-Power Millimeter-Wave Helix TWTs. IEEE Trans. Plasma Sci. 2010, 38, 1227-1238. [CrossRef]

2. $\quad$ Liu, L.; Wei, Y.; Shen, F.; Zhao, G.; Yue, L.; Duan, Z.; Wang, W.; Gong, Y.; Li, L.; Feng, J. A Novel Winding Microstrip Meander-Line Slow-Wave Structure for V-Band TWT. IEEE Electron Device Lett. 2013, 34, 1325-1327. [CrossRef]

3. Luo, J.; Feng, J.; Gong, Y. A Review of Microwave Vacuum Devices in China: Theory and Device Development Including High-Power Klystrons, Spaceborne TWTs, and Gyro-TWTs. IEEE Microwave. Mag. 2021, 22, 18-33. [CrossRef]

4. Paoloni, C.; Gamzina, D.; Letizia, R.; Zheng, Y.; Luhmann, N.C., Jr. Millimeter wave traveling wave tubes for the 21st Century. J. Electromagn. Waves Appl. 2021, 35, 567-603. [CrossRef]

5. Wang, Z.; Wang, H.; Xu, D.; He, T.; Li, X.; Gong, H.; Lu, Z.; Duan, Z.; Wei, Y.; Gong, Y. Study on Planar Slow Wave Structure of Traveling Wave Tube. Vac. Electron. 2018, 2, 20-30.

6. Chua, C.; Aditya, S. A 3-D U-Shaped Meander-Line Slow-Wave Structure for Traveling-Wave-Tube Applications. IEEE Trans. Electron Devices 2013, 60, 1251-1256. [CrossRef]

7. Chua, C.S.; Aditya, S.; Shen, Z.X. Planar Helix with Straight-Edge Connections in the Presence of Multilayer Dielectric Substrates. IEEE Trans. Electron Devices 2010, 57, 3451-3459. [CrossRef]

8. Chua, C.; Tsai, J.M.; Aditya, S.; Tang, M.; Ho, S.W.; Shen, Z.; Wang, L. Microfabrication and Characterization of W-Band Planar Helix Slow-Wave Structure with Straight-Edge Connections. IEEE Trans. Electron Devices 2011, 58, 4098-4105. [CrossRef]

9. Chua, C.S.; Tsai, M.L.J.; Tang, M.; Aditya, S.; Shen, Z.X. Microfabrication of a Planar Helix with Straight-Edge Connections Slow-Wave Structure. Adv. Mater. Res. 2011, 254, 17-20. [CrossRef]

10. Shen, F.; Wei, Y.; Xu, X.; Liu, Y.; Huang, M.; Tang, T.; Gong, Y. U-shaped microstrip meander-line slow-wave structure for Ka-band traveling-wave tube. In Proceedings of the 2012 International Conference on Microwave and Millimeter Wave Technology (ICMMT), Shenzhen, China, 5-8 May 2012.

11. Shen, F.; Wei, Y.; Yin, H.; Gong, Y.; Xu, X.; Wang, S.; Wang, W.; Feng, J. A Novel V-Shaped Microstrip Meander-Line Slow-Wave Structure for W-band MMPM. IEEE Trans. Plasma Sci. 2011, 40, 463-469. [CrossRef]

12. Shen, F.; Wei, Y.; Xu, X.; Lai, J.; Huang, M.; Zhao, G.; Gong, Y. Rhombus-shaped Microstrip Meander-line Slow-wave Structure for $140 \mathrm{GHz}$ Traveling-wave Tube. In Proceedings of the 2012 IEEE International Vacuum Electronics Conference (IVEC), Monterey, CA, USA, 24-26 April 2012.

13. Lu, Z.; Ge, W.; Wen, R.; Wang, Z.; Gong, H.; Wei, Y.; Gong, Y. 0.2-THz Traveling Wave Tube Based on the Sheet Beam and a Novel Staggered Double Corrugated Waveguide. IEEE Trans. Plasma Sci. 2020, 48, 3229-3237. [CrossRef]

14. Shao, W.; Tian, H.; Wang, Z.; Lu, Z.; Gong, H.; Tang, T.; Duan, Z.; Wei, Y.; Gong, Y.; Feng, J. Study for 850 GHz Sheet Beam Staggered Double-Vane Traveling Wave Tube Considering the Metal Loss. In Proceedings of the 2018 IEEE International Vacuum Electronics Conference (IVEC), Monterey, CA, USA, 21 April 2018.

15. Xu, D.; Shao, W.; He, T.; Wang, H.; Wang, Z.; Lu, Z.; Gong, H.; Duan, Z.; Feng, J.; Gong, Y. Investigation on 0.1 THz Array Beams Folded Waveguide Traveling Wave Tube. In Proceedings of the 2019 Photonics \& Electromagnetics Research Symposium (Piers), Xiamen, China, 17-20 December 2019. 
16. Ding, C.; Wei, Y.; Wang, Y.; Xu, J.; Tang, T.; Huang, M.; Zhang, L.; Li, Q.; Xia, L.; Gong, Y.; et al. 2-Dimensional Microstrip Meander-line for Broad Band Planar TWTs. In Proceedings of the 2016 IEEE International Vacuum Electronics Conference (IVEC), Monterey, CA, USA, 19-21 April 2016.

17. Wang, S.; Aditya, S.; Xia, X.; Ali, Z.; Miao, J. On-Wafer Microstrip Meander-Line Slow-Wave Structure at Ka-Band. IEEE Trans. Electron Devices 2018, 65, 2142-2148. [CrossRef]

18. Wang, S.; Aditya, S.; Xia, X.; Ali, Z.; Miao, J.; Zheng, Y. Ka-Band Symmetric V-Shaped Meander-Line Slow Wave Structure. IEEE Trans. Plasma Sci. 2019, 47, 4650-4657. [CrossRef]

19. Ryskin, N.M.; Rozhnev, A.G.; Starodubov, A.V.; Serdobintsev, A.A.; Pavlov, A.M.; Benedik, A.I.; Torgashov, R.A.; Torgashov, G.V.; Sinitsyn, N.I. Planar Microstrip Slow-Wave Structure for Low-Voltage V-band Traveling-Wave Tube with a Sheet Electron Beam. IEEE Electron Device Lett. 2018, 1, 5. [CrossRef]

20. He, T.; Li, X.; Wang, Z.; Wang, S.; Lu, Z.; Gong, H.; Duan, Z.; Feng, J.; Gong, Y. Design and Cold Test of Dual Beam Azimuthal Supported Angular Log-Periodic Strip-Line Slow Wave Structure. J. Infrared Millimeter Terahertz Waves 2020, 41, 785-795. [CrossRef]

21. Wang, H.; Wang, Z.; Li, X.; He, T.; Xu, D.; Gong, H.; Tang, T.; Duan, Z.; Wei, Y.; Gong, Y. Study of a miniaturized dual-beam TWT with planar dielectric-rods-support uniform metallic meander line. Phys. Plasmas 2018, 25, 63113. [CrossRef]

22. Dong, Y.; Chen, Z.; Li, X.; Wang, H.; Wang, Z.; Wang, S.; Lu, Z.; Gong, H.; Duan, Z.; Feng, J.; et al. Ka-band dual sheet beam traveling wave tube using supported planar ring-bar slow wave structure. J. Electromagn. Waves Appl. 2020, 34, 2236-2250. [CrossRef]

23. Wang, H.; Wang, S.; Wang, Z.; Li, X.; Xu, D.; Duan, Z.; Lu, Z.; Gong, H.; Aditya, S.; Gong, Y. Dielectric-Supported Staggered Dual Meander-Line Slow Wave Structure for an E-Band TWT. IEEE Trans. Electron Devices 2021, 68, 369-375. [CrossRef]

24. Wang, Y.; Dong, Y.; Wang, S.; Gong, Y. Dielectric-Supported Rhombus-Shaped Meander-Line Slow-Wave Structure for a V-band Dual-Sheet Beam Traveling Wave Tube. In Proceedings of the 2021 Photonics \& Electromagnetics Research Symposium (Piers), Hangzhou, China, 21-25 December 2021.

25. Xu, D.; Wang, H.; He, T.; Li, X.; Lu, Z.; Gong, H.; Wang, Z.; Duan, Z.; Gong, Y. Study on Broadband Ridge-Loaded Symmetrical Conformal Microstrip Meander Line Traveling Wave Tube at Ka-Band. In Proceedings of the 2019 International Vacuum Electronics Conference (IVEC), Busan, Korea, 28 April 2019.

26. Wang, H.; Wang, S.; Wang, Z.; Li, X.; He, T.; Xu, D.; Duan, Z.; Lu, Z.; Gong, H.; Gong, Y. Study of an Attenuator Supporting Meander-Line Slow Wave Structure for Ka-Band TWT. Electronics 2021, 10, 2372. [CrossRef]

27. Zhao, C.; Aditya, S.; Wang, S.; Miao, J.; Xia, X. A Wideband Microfabricated Ka-Band Planar Helix Slow-Wave Structure. IEEE Trans. Electron Devices 2016, 63, 2900-2906. [CrossRef]

28. Introduction of CST Microwave Studio. Available online: https:/ /www.cst.com/products/cstms (accessed on 1 December 2016).

29. Wang, S.; Zhao, C.; Aditya, S.; Chua, C. Thermal Characteristics of a Ka-Band Planar Helix Slow-Wave Structure. In Proceedings of the 2015 International Vacuum Electronics Conference (IVEC), Beijing, China, 27-29 April 2015. 